\title{
La fiabilidad teórica del determinismo Un examen desde la propuesta de Mariano Artigas
}

The theoretical reliability of determinism. A review from the proposal of Mariano Artigas

\author{
MARTÍN MONTOYA \\ Universidad de Navarra \\ jmontoya.1@unav.es
}

Resumen. El presente artículo tiene dos finalidades. La primera es demostrar que la teoría del determinismo, que pretende fundarse en los principios de las ciencias experimentales, no puede ser considerada como una explicación compatible con tales ciencias. Para ello, utilizamos algunas ideas de Mariano Artigas sobre la capacidad explicativa de las teorías científicas y sobre su fiabilidad presentes en su libro La mente del universo. Con esto, buscamos alcanzar el segundo objetivo: mostrar la importancia de la obra de Artigas para la disolución de complejos debates contemporáneos, como es el caso de la actual discusión sobre la existencia de la libertad humana.

Palabras clave: causalidad; ciencias experimentales; explicación; libertad humana.

Abstract. This article has two purposes. The first is to demonstrate that the theory of determinism, which claims to be based on the principles of experimental science, cannot be considered as an explanation compatible with such sciences. To do this, we use some ideas of Mariano Artigas on the explanatory power of scientific theories and their reliability from his book The Mind of the Universe. Through this process we seek to achieve the second objective: to show the importance of the work of Artigas to dissolve complex contemporary debates, such as the current discussion about the existence of human freedom.

Keywords: causation; empirical sciences; explanation; human freedom. 


\section{La ciencia experimental y los presupuestos del determinismo}

La dinámica del debate sobre la existencia del libre albedrío se encuadra en la discusión entre las antagónicas posturas sobre la compatibilidad y la incompatibilidad entre el libre albedrío y el determinismo ${ }^{1}$. Por un lado, se tiene a los compatibilistas ${ }^{2}$, por el otro, a libertaristas y deterministas. El compatibilismo ha hecho grandes esfuerzos por demostrar que es posible admitir un cierto determinismo en el comportamiento del agente, y que esta afirmación es compatible con la libertad y/o con la responsabilidad moral (Sellars 1966; Frankfurt 1969; Fischer 2010) ${ }^{3}$. El determinismo niega esta tesis.

Los deterministas pueden afirmar su posición de dos formas. La primera es que podemos alcanzar una explicación completa de los sucesos de la realidad, incluido el comportamiento humano, una vez que hayamos obtenido una comprensión científica de las leyes del mundo y los hechos del pasado. Este tipo de determinismo asume que todos los eventos de los que se compone el mundo están necesariamente relacionados de un modo causal (Wiggins 1987, 725; Van Inwagen 1983, 65). Para el determinismo no caben explicaciones de la realidad diversas de las que se pueden ofrecer desde la lógica proposicional (Hintikka 1973; 1979) o desde las ciencias

1 Una buena sistematización de los argumentos implicados en este debate se encuentra en (Vihvelin 2011).

2 La idea general del compatibilismo es la que define John Martin Fischer como la postura en la que "una noción central de la libertad y otra genuina y robusta de la responsabilidad moral, son compatibles con la doctrina del determinismo causal” (Fischer 2007, 44). Al hablar de una noción central de libertad, Fischer se refiere a la noción de libertad que sea fundamento para el concepto de responsabilidad moral que se aplica a la ética, la psicología, la jurisprudencia, etc. No se refiere, por tanto, a la libertad social, política, o moral, sino a una idea de libertad que pueda ser soporte de todas ellas. Por otro lado, la noción genuina y robusta de responsabilidad moral hace referencia a la idea de imputabilidad básica de la culpa o del mérito. La noción será genuina si esta versa únicamente sobre la idea de asignación de la responsabilidad por las consecuencias de las acciones; y será robusta, si no admite refutaciones.

3 En estas líneas no voy a realizar ningún análisis del compatibilismo. Considero que es una tarea que excede la finalidad de este artículo. Además, para analizar el compatibilismo se requiere la introducción de algunos conceptos y posturas contemporáneas sobre la libertad que podrían desviar nuestra atención. Considero suficiente mostrar, de momento, una visión general del contraste entre el determinismo y el libertarismo. 
experimentales pero enfocadas bajo la lente de un rígido esquema causal, donde el efecto se sigue necesariamente de su causa (Hoefer 2015). Para los deterministas bastaría simplemente con esperar que las ciencias experimentales pudieran dar una explicación definitiva de los componentes de la realidad, y de sus leyes, para poder alcanzar una máxima predictibilidad del futuro (Van Inwagen 1983, 16). El comportamiento individual de los agentes estaría también implicado en tal proyección de los eventos.

La segunda posición del determinismo actual arroja más luz a esta cuestión. Esta postura sigue afirmando que el sujeto es analizable desde la objetividad de las ciencias experimentales, y que tal sujeto está sumido en un tipo restrictivo de causalidad que enlaza necesariamente los eventos, que funcionan como causas y efectos. Sin embargo, la perspectiva es cognitiva. Desde este punto de vista, los individuos pueden estar determinados de un modo conductista por los eventos que componen el mundo aunque estos no estuviesen regidos por leyes de tipo determinista (Van Inwagen 1998, 370). Es decir, aún cuando pudiéramos suponer la indeterminación causal de los eventos del mundo, no sería valido aceptar a continuación que el agente puede verse libre de una determinación causal, sea por factores internos de su propia consciencia o por factores externos. Para el determinismo, si aceptamos que el conocimiento puede establecer relaciones causales entre los eventos de un modo necesario para generar información relevante para el sujeto, entonces nos debemos ver obligados a indicar que tales conexiones son necesarias.

Las propuestas deterministas afirman que esto debe suponerse ya que, por un lado, si el conocimiento humano no puede establecer relaciones causales consistentes, entonces es difícil considerar que dicho conocimiento pueda organizar adecuadamente la información que recibe. Y, por el otro lado, negar tales relaciones causales llevaría a una especie de caos cognitivo del agente que lo dejaría a merced de la ocurrencia azarosa de los eventos del mundo. Por tanto, el agente no podría poseer un control sobre las ocurrencias relacionadas con su comportamiento, debido a la carga de necesidad que representa la relación causal implicada en esta explicación de su conocimiento. Es decir, para el determinismo, o indicamos que el agente 
no puede impedir el establecimiento de tales conexiones causales entre su conocimiento y la información que recibe, o caso contrario tendremos que indicar que el agente se encuentra a merced del azar. . A esto último se le ha llamado el problema de la suerte (Campbell 2011, 23).

El determinismo se apoya en una lectura fisicalista ${ }^{5}$ de la mente humana y de los avances de neurocientíficos ${ }^{6}$, que no buscan dar ese paso hacia el determinismo, como es el caso de Patricia Churchland. Su propuesta se ha basado, en varios de sus escritos (Churchland 2011; 2013; Suhler \& Churchland 2011), en una revisión de los términos con los que nos referimos a fenómenos como “creencia”, "conciencia” y "voluntad libre”, los cuales

4 En la perspectiva de este debate, el problema del control de las acciones no se resuelve apelando a la voluntad del agente. Para casi la totalidad de las posturas hablar de voluntad es introducir un dualismo de mente y voluntad, o mente y cuerpo. Otro problema distinto al del dualismo se da al hablar de la trazabilidad de las acciones, esto es, a la posibilidad de remontarse en la cadena de sucesos causales hasta llegar al origen de la acción. Pero esta cuestión cae en una paradoja insoluble si es que se mantiene que la voluntad se compone de deseos e intenciones que se deben remontar hasta algunos deseos no plenamente conscientes del agente. Como ha indicado Campbell (2011, 32-33), siguiendo a Aristóteles, este problema es superfluo si se consideran las condiciones epistémicas adecuadas para la responsabilidad moral sabiendo que siempre dependemos de algo que está a nuestra disposición para actuar, sea conocido o no. Tales condiciones son que no se dé una ignorancia sobre las particularidades de la acción y que, si se da tal ignorancia, esta no sea culpable, tal como afirma también Zimmerman (1997, 423).

5 El fisicalismo es "un programa unificador de tipo metafísico y epistemológico guiado por la idea de que todo es una manifestación de los aspectos físicos de la existencia” (Liz 2007, 97). La idea central de esta postura es que todo lo que existe puede llegar a pertenecer al dominio de la ciencia física. Esto incluye hechos o sucesos, seres animados e inanimados, tanto si pertenecen a ciencias empíricas como la química o la biología, como a otras no clasificables en tal ámbito como la psicología. Me quedé aquí, continuar revisión de notas

6 Esta indicación puede apreciarse a través de interpretaciones deterministas de los resultados del experimento neurológico de Libet (Libet, Gleason, Wright \& Pearl 1983). En este experimento se midió el tiempo de respuesta de una acción después de un estímulo, siguiendo los parámetros de la actividad cerebral. El resultado presentó un desfase temporal de un tercio de segundo entre el estímulo y el inicio de la actividad consciente del agente. La aparición de esta actividad cerebral no consciente llevó a algunos autores, como Daniel Wegner (2002), a afirmar la existencia de factores deterministas en el cerebro que condicionan las decisiones futuras del agente, dejando un muy poco "espacio" para la aparición de la libertad de la conciencia. Por tanto, según Wegner, la libertad es una ilusión. Su lectura del experimento de Libet no toma en cuenta la dimensión espiritual del ser humano que puede arrojar interpretaciones distintas de los mismos resultados (Murillo y Giménez-Amaya 2008; Alexander et al. 2015). 
pueden ser -según su visión- explicados científicamente desde el funcionamiento del cerebro humano. Por tanto, para Churchland, los términos que se emplean para hablar de la libertad y la conciencia pueden ser reducidos a las explicaciones experimentales de la neurociencia, la cual podría alcanzar en el futuro una comprensión total de tales funcionalidades cerebrales y, de este modo, dar un sentido científico a dichos términos. Churchland expresa que hay malentendidos sobre el tipo de control que el agente tiene sobre su comportamiento. Estos se deben a una mala comprensión del papel que tienen los procesos no conscientes en el conocimiento, $\mathrm{y}$ un desconocimiento de su función como motor de las habilidades sociales: el agente solo puede tener responsabilidad sobre aquellos procesos conscientes en los que tenga competencia normativa y ejecutiva.

Un ejemplo que podemos mencionar de la dirección que desea tomar Churchland se da en las habilidades artísticas -como tocar el piano o el violín-, que se presentan como una especie de automatismos sobre los que el agente posee un cierto control. Pero la propuesta de Churchland se dirige más hacia la descripción de las condiciones neurológicas requeridas para que estos procesos funcionen. De hecho, para la neurofilósofa la reducción de las explicaciones del comportamiento de las personas a sus funciones cerebrales debe llevar a una comprensión de la responsabilidad moral de los seres humanos sin necesidad de indicar la existencia de algo así como la "conciencia".

Las propuestas de autores como Churchland no son deterministas. Sin embargo, la idea de que la tarea científica debe comprenderse como la búsqueda de una explicación totalizante de los fundamentos de la realidad abre la puerta a las interpretaciones deterministas. El objetivo del determinismo es encontrar una especie de "mecanismo" que en su aplicación hermenéutica a los fenómenos del mundo pueda ofrecernos la certeza del porqué de la ocurrencia de los eventos. En propuestas como la de Churchland se trata de explicar el sentido de los términos con los que comprendemos el comportamiento humano, teniendo como paradigma de interpretación los mecanismos de funcionamiento del cerebro. Esto es usado por el determinismo para invalidar el significado de los términos 
asociados a la libertad en las explicaciones filosóficas, y desestabilizar la intuición ordinaria de que somos seres libres. Como una cuestión derivada de la anterior, se busca poner en duda la experiencia humana de la libertad partiendo de la idea de la ciencia experimental como único saber sobre la realidad física. Finalmente, las posturas deterministas suelen introducir la idea de causalidad ya indicada, que es evidentemente apriorística, en el sentido de que es una concepción previa a la experiencia real de las ciencias experimentales. Desde este punto de vista, para el determinismo la totalidad de las ciencias deberá encajar en tal esquema conceptual por la exigencia de la coherencia y unificación de las explicaciones de la realidad. Este es el componente lógico a priori de esta propuesta, que busca ser el fundamento de todas las experiencias de esa pretendida ciencia omniabarcante.

Para entender por qué el determinismo sigue vigente como consideración teórica hay dos respuestas. La primera es la presencia de la idea, en estos debates, de que aunque no tenemos ninguna buena razón para creer que el determinismo es verdadero, tampoco tenemos ninguna buena razón para creer que es falso, y por tanto, aún podría ser cierto que estamos determinados causalmente, aunque sea a un nivel subatómico (Balaguer 2009a, 21). La primera estrategia del determinismo, por tanto, es instalar la duda sobre nuestra propia experiencia de la libertad, la cual adquirimos en el desarrollo de cualquier actividad humana.

La segunda razón se basa en la estrategia determinista por la que, una vez instalada la duda, se indica que la libertad no es explicable desde el nivel de las ciencias empíricas (Balaguer 2009b). La idea que maneja el determinismo para fundamentar esta afirmación es que los procesos humanos de toma de decisiones que presuponen la libertad no son compatibles con un mundo de condiciones empíricas explicado por las ciencias experimentales. Por tanto, lo que indican las posturas que van en contra de la existencia del libre albedrío es que la mera presencia de fuerzas ajenas al sujeto puede ser considerada como una especie de determinismo, por efecto de la relación causal necesaria que resultaría de unir voluntad y razón ${ }^{7}$, o razones reactivas

7 Esta idea ha sido adoptada en la tradición de la filosofía que se desarrolla en gran parte de los Estados Unidos desde los planteamientos de Thomas Hobbes. Este indica que la volun- 
y acciones ${ }^{8}$, etc. En este caso, la libertad estaría considerada como una noción antagónica a la idea de causalidad, la cual se asimila a un tipo de causa a priori aplicada a un mundo meramente físico ${ }^{9}$. La postura del determinismo es lógico-fisicalista, y se fundamenta en un esquema epistemológico que se aplica de modo a priori al conocimiento que pueden ofrecer las diversas ciencias experimentales.

En las siguientes líneas no desarrollaré un análisis de la noción de causalidad implicada en este debate filosófico puesto que esto derivaría en consideraciones metafísicas que no son el objeto de este artículo. En cambio, buscaré mostrar cómo es que una comprensión adecuada de la tarea de las ciencias experimentales y su fiabilidad puede ayudar a demostrar que los presupuestos epistemológicos de la estrategia determinista, que indico a continuación, son inconsistentes. La estrategia del determinismo para convertirse en una postura fiable en cuanto a su capacidad explicativa se basa en:

(a) la introducción de una duda como primer principio epistemológico. Se trata de una duda sobre la libertad humana, teniendo como punto de partida las dificultades de hacerla compatible con la causalidad

tad no es más que una capacidad apetitiva básica. Capacidad de apetitos, de deseos, que los humanos y los animales pueden mostrar perfectamente por igual. La diferencia entre hombres y animales, radica simplemente en la mayor complejidad intelectiva de los seres humanos. No habría actos voluntarios o decisiones, sino simplemente respuestas pasivas ante los estímulos. Como ha indicado Thomas Pink (2004, 55-65), Hobbes sitúa las acciones posibles enteramente fuera del verdadero dominio de la voluntad. La acción no ocurre como un ejercicio voluntario del agente que decide ante los motivos que el intelecto le presenta, sino como un efecto producido por impulsos que conducen al agente hacia lo deseado. De este modo la libertad puede definirse simplemente como la inexistencia de obstáculos para la satisfacción de los deseos.

8 La noción original de razones reactivas se puede encontrar en (Strawson 1962).

9 El determinismo absoluto está lejos de haber sido abandonado. Este tipo de determinismo es la postura teórica más convincente para algunos autores que lo defienden como una propuesta real ante la ineficacia de las diferentes teorías para explicar la libertad de la acción (Honderich 2011, 442-456). Lo que esperan posturas como estas es que se demuestre que la duda en contra de la libertad es falsa pero bajo condiciones que presuponen el determinismo. Esto es, que se identifique la libertad de los agentes en un escenario donde se asume que los procesos cognitivos del sujeto, al involucrar una relación con el mundo físico, están determinados. 
lógico-fisicalista que se pretende introducir como fundamento hermenéutico de la explicación en la ciencia experimental;

(b) el paso desde la duda sobre la existencia de la libertad hasta la certeza de su inexistencia. Esto se lleva a cabo a través de la reafirmación del tipo de causalidad asumido en (a), sin el debido examen de su verdadera capacidad para explicar la realidad. El tipo de causalidad supuesto por el determinismo para interpretar la realidad se erige como paradigma para la explicación de los fenómenos del mundo;

(c) se concluye lo que se ha supuesto, es decir, que no es posible afirmar que los seres humanos son libres, ya que la libertad no es compatible con una visión científica del mundo tal como la describen las posturas deterministas.

Para los fines de este artículo me concentraré en (b), el paso de la duda sobre la existencia de la libertad a la certeza de su inexistencia. En este punto se manifiestan con mayor claridad las limitaciones teóricas del determinismo, que pretende dar cuenta de la realidad sin más principios explicativos que la causalidad lógico-fisicalista. Pero antes, mostraré cómo el libertarismo metafísico contemporáneo ha fallado en sus intentos de explicar la libertad por no poder superar la barrera lógico-fisicalista impuesta por el determinismo. De este modo, se podrá comprender que las respuestas adecuadas para alcanzar un cierto progreso en este debate van en la línea de un cambio radical en el paradigma explicativo. Además, en el siguiente apartado pondré a prueba la fiabilidad de la dinámica explicativa del determinismo a través de algunas ideas que Mariano Artigas ha vertido en su libro La mente del universo.

\section{Libertarismo, explicación y fiabilidad científica}

La postura antagónica al determinismo es el libertarismo metafísico. Este busca refutar las pretensiones teóricas del determinismo y dar soluciones al enclaustramiento lógico-fisicalista de esta posición. El libertarismo metafísico se ha dividido en tres posturas, de las cuales consideraremos las 
dos más importantes ${ }^{10}$. La primera es el libertarismo causal del agente, que afirma que las acciones de una persona libre y responsable son causadas por ella misma como una sustancia irreducible o no compuesta de eventos. Esta afirmación es sostenida por filósofos como Timothy O’Connor (2011; 2014). Este autor argumenta que si tomamos metafísicamente en serio las afirmaciones de que la mente humana está compuesta de materia, que la causalidad es real, y que el mundo se compone de sustancias y no de eventos, entonces es posible decir que el sujeto causa sus acciones libremente. A la posición de O’Connor se le ha criticado por no tener una definición general de sustancia que sea lo suficientemente consistente para diferenciarla de los eventos (Kane 2014, 202-206).

La objeciones pueden ser aún más efectivas, y más provechosas para lo que intentamos explicar en este artículo. O’Connor no indica cómo se especifica el tipo de sustancia libre que, según él, es la persona. Es decir, para evitar las objeciones del determinismo, O’Connor evade la explicación sobre quién es el agente, o qué elementos participan en la explicación de la acción humana. Pero siguiendo esta estrategia es difícil comprender en qué puede diferenciarse la persona humana de los demás elementos del mundo. La argumentación de O’Connor se lleva a cabo con la evidente intención de evitar la suposición de que el agente está compuesto de partes, a las que se pueda aplicar el rígido esquema causal determinista. Por supuesto que si el sujeto no está compuesto de “partes”, entonces no será posible establecer ningún tipo de relación entre ellas. Pero, siguiendo esta estrategia argumentativa, O’Connor olvida la explicación sobre el agente y su conocimiento, sobre su entorno y lo que le mueve a actuar, concediendo cierta validez al esquema causal lógico-fisicalista del determinismo, como principio hermenéutico que podría llenar el vacío de esta interpretación del libertarismo. Al final de cuentas, lo único que consigue es evitar el problema de la explicación de la libertad del agente, manteniendo la validez de la postura que busca refutar.

10 La tercera postura es el libertarismo no causal. Esta es una posición crítica ante las posturas que observaremos, pero no presenta una propuesta consistente sobre el modo de realizar una acción de un modo no causal. 
De este modo, sin pretenderlo, el libertarismo de O'Connor se mantiene en el mismo paradigma explicativo que el determinismo.

La crítica contra O'Connor cobra fuerza si consideramos que, al carecer de una explicación del modo en que se realizan las acciones, este autor ha propuesto que la libertad emerge de la sustancia material (O’Connor 2002). $\mathrm{Si}$ la persona es considerada epistemológicamente como una sustancia asimilable a otras sustancias físicas entonces la libertad, en la postura de O'Connor, solo puede derivar causalmente desde la materia. El emergentismo podría ser considerado como un postulado a priori, sin elementos que separen la explicación del paradigma determinista de la causalidad y potencien la comprensión de la experiencia de la libertad en las actividades humanas.

La segunda postura es el libertarismo de la causalidad de los eventos. Esta afirma que las acciones de una persona libre y responsable son causadas por eventos o estados de la mente humana, pero que existe algún grado de indeterminación en la causalidad de las acciones con respecto a tales eventos previos. Esta postura es defendida por Robert Kane. Este autor habla de acciones autoformadas que configuran la mente humana, pero que están a disposición del agente que posee la responsabilidad última por su generación y utilización (Kane 2007). La postura de Kane es bastante articulada, y a través de ella pueden afirmarse ciertas disposiciones del agente para ponderar reflexivamente sus acciones, y hacer posible una cierta independencia del sujeto ante los eventos que componen su entorno.

Algunos críticos de la postura de Kane, como Randolph Clarke y Carl Ginet, indican que asumir este tipo de disposiciones lleva al libertarismo a una posición demasiado cercana al determinismo, aún cuando este sea muy débil (Clarke 2014). Ginet afirma que aunque la postura de Kane pueda brindar una explicación a la libertad con este tipo de capacidades, su teoría no llega a asegurar la libertad de las acciones que no están alineadas operativamente con tales capacidades (Ginet 2014). Desde la perspectiva de Ginet, la postura de Kane podría ser susceptible de la siguiente objeción: De acuerdo con Kane, las acciones libres del agente serían aquellas realizadas por él cuando reflexiona sobre la información recopilada de su entorno (la cual precede a la causalidad de sus acciones). Sin embargo, para Ginet, esto 
no podría ocurrir para las acciones que no son objeto de tal proceso consciente de reflexión. Por tanto, Ginet indica que la postura de Kane permite postular una relación causal necesaria entre los procesos no conscientes del sujeto y la información que este recopila de su entorno. Desde este punto de vista, libertad y determinismo podrían estar coexistiendo en la misma postura. Por tanto, sostienen sus detractores, esta propuesta roza el compatibilismo y coloca al libertarismo metafísico en una encrucijada insuperable. El libertarismo se encontraría dentro del cerco tendido por los presupuestos del determinismo, puesto que mantiene el mismo paradigma causal de explicación. Es decir, siguiendo los principios epistemológicos del determinismo:

(a') se introduce una duda sobre el libertarismo. El libertarismo pierde fiabilidad al dar cierta validez a la causalidad lógico-fisicalista. Esto ocurre porque el libertarismo no cambia de paradigma para explicar la libertad (no lleva a cabo ninguna explicación sobre la identidad del agente que actúa), sino que simplemente evita que la causalidad entre en las explicaciones sobre la acción humana y la libertad;

(b') los objetores deterministas pasan desde la duda hasta la certeza sobre la invalidez del libertarismo: suponiendo (a'), el libertarismo queda sin fundamentos teóricos para refutar los intentos de las posturas deterministas de introducir la causalidad lógico-fisicalista como principio hermenéutico válido para explicar la acción humana;

(c') con este tipo de argumentación, el libertarismo cede protagonismo teórico, de modo indirecto, ante la afirmación de que la visión causal lógico-fisicalista del determinismo podría servir como paradigma científico para explicar el mundo.

El debate sobre la existencia del libre albedrío, como hemos observado, hunde sus raíces en la idea de que la explicación de las ciencias experimentales, tal como la entienden las diversas posturas deterministas, es suficiente para alcanzar un conocimiento fiable del mundo. Pero, además, que el esquema de las ciencias se debe regir por los parámetros lógico-fisicalistas explicados a lo largo de estas líneas. Esta idea es clara en el determinismo, pero alcanza también a posturas como el libertarismo, que se mantienen 
dentro del mismo paradigma de explicación de la realidad. Pasemos ahora al examen de la fiabilidad del determinismo.

Mariano Artigas propone su propia explicación de la fiabilidad dentro de las ciencias en su libro La mente del universo. Su finalidad es evitar interpretaciones escépticas y relativistas sobre la construcción de las teorías científicas. Artigas indica que la idea de fiabilidad es aplicable en el ámbito de la epistemología cuando queremos decir que determinadas construcciones científicas sirven para alcanzar los objetivos de la empresa científica. Para ello las teorías científicas, o -en mi opinión- aquellos proyectos interdisciplinares que buscan ser compatibles con los datos que ofrecen las ciencias en general, deben expresarse y comprobarse de modo intersubjetivo. Además, la fiabilidad de una teoría que busca estar en concordancia con los métodos de las ciencias requiere que las pruebas para su validez impliquen contrastaciones empíricas. Y, finalmente, una teoría es fiable si puede conducir a nuevas predicciones, y que estas representen un progreso en el desarrollo de la ciencia (Artigas 1999, 313-314).

Considero que los principales problemas de la dinámica explicativa del determinismo se pueden disolver a través de un examen apoyado en el primero de los aspectos de la fiabilidad, es decir, su intersubjetividad. Esto se debe a dos razones. La primera es que los aspectos del control experimental o contrastabilidad empírica, el poder predictivo y el progreso, pertenecen propiamente a las ciencias experimentales. Por otro lado, la dinámica explicativa del determinismo busca pertenecer al ámbito filosófico manteniendo una cierta relación teórica con los principios de las ciencias empíricas. Por tanto, al verse privado de la posibilidad de asentarse en el ámbito de las ciencias experimentales a través de los otros tres aspectos, si pretende mantener una cierta relevancia teórica, el determinismo no puede renunciar a la intersubjetividad. Caso contrario, podremos concluir que esta postura es un simple constructo ideal, vacío de todo contenido que le pueda asociar a la realidad y, por tanto, que no puede sostener ninguna visión científica del mundo.

Artigas indica que la intersubjetividad se basa en la aceptación de reglas que surge del hecho objetivo de que existen pautas naturales ordenadas, 
y del hecho subjetivo de que es posible introducir estipulaciones que hacen posible establecer lenguajes intersubjetivos (Artigas 1999, 314). La dinámica explicativa de las ciencias no se basa simplemente en afirmar un orden natural regido por leyes objetivas que no permiten un cambio en las estimaciones. Por el contrario, el elemento subjetivo es una pieza clave en el avance y progreso de las ciencias. Sin intersubjetividad no hay posibilidad de comunicación de los resultados obtenidos en la experimentación, y sin esta comunicabilidad la empresa científica no puede tener éxito. Los resultados en las ciencias experimentales no se imponen por su simple evidencia, sino que obedecen a modelos previamente discutidos y aprobados, y esta es una dinámica fundamental que permite el progreso científico, el cual no se debe solo al poder predictivo y el control experimental, sino también a la explicitación de factores convencionales que permiten aplicar determinados principios a diversos contextos (Artigas 1999, 271-272).

La noción de intersubjetividad es importante tanto en su elemento objetivo como en el subjetivo, en cuanto que atiende el orden de lo natural e introduce con el elemento subjetivo la capacidad humana de obrar dentro de la misma actividad científica. Las ciencias progresan sobre la base de las actividades humanas, de las cuales la ciencia misma debe ser considerada como una empresa de conocimiento que no puede verse reducida solo al ámbito de lo objetivo. Con esto es posible evitar la indiscriminada aplicación a priori de esquemas lógicos que vayan en contra de la capacidad del científico para pensar en la oportunidad de introducir nuevos modelos que mejoren las explicaciones de los fenómenos.

Para Artigas, siguiendo a Hübner (Artigas 1999, 271), en el mundo de las ciencias todos los enunciados científicos dependen de preceptos a priori. Así, por ejemplo, el enunciado meta-científico del tipo: "si suponemos un cierto grupo de enunciados a priori, obtendremos determinados resultados empíricos" no es en sí mismo a priori, ya que la relación condicional como tal se aplica a determinados contextos en los cuales puede explicarse un mismo fenómeno de una forma o de otra (Artigas 1999, 271). Hay diversos paradigmas en las ciencias, pero ninguno de ellos sigue un esquema preconcebido para todas ellas. Sin embargo, la pretensión del determinismo es 
postular la causalidad como un esquema meta-científico, aplicable a toda la realidad. Por tanto, la afirmación de la causalidad lógico-fisicalista como un principio de las ciencias, que puede poner en duda las intuiciones subjetivas y ordinarias de la experiencia de la libertad, se ve refutada por la misma dinámica de las ciencias experimentales. Esta incluye unos factores subjetivos y de convencionalidad que no pueden ser absorbidos dentro un único principio cuya aplicación indiscriminada pretenda llevar a un conocimiento objetivo del mundo, cuando en realidad lo único que consigue es recortar elementos fundamentales del mismo, como es el caso de la libertad humana.

El valor de los enunciados científicos depende de contextos que incluyen estipulaciones teóricas y pragmáticas; por tanto, la objetividad que se puede alcanzar en las ciencias es siempre contextual (Artigas 1999, 271). Para Artigas esto no implica ninguna arbitrariedad. Una vez que se establece un conjunto específico de estipulaciones, y se utilizan para definir una objetivación en un determinado contexto, se acota la libertad para determinar el valor de las construcciones que se pueden formular en ese ámbito de la realidad (Artigas 1999, 272). Sin embargo, dado que los principios a priori sobre los que se apoya el progreso de la ciencia no pueden sucederse indefinidamente, hay que estimar que estos surgen en concordancia con la situación histórica en la que son descubiertos. Este modo de estimar los principios a priori sobre los que se realizan las estipulaciones en el mundo de la ciencias hace que a estas les preceda no un esquema lógico sobre el que deberán encajar para mantener una unidad, sino la actividad propiamente humana de la búsqueda de la verdad científica. Las ciencias experimentales se apoyan en el uso y la experiencia previa que remite a nuevos esquemas de un modo no necesario. Las ciencias no se rigen por esquemas a priori, y por tanto, la idea de que la visión científica del mundo requiere apelar únicamente a explicaciones causales para alcanzar un conocimiento coherente de la realidad debe ser abandonada en este debate.

La afirmación de un principio causal lógico-fisicalista que impide explicar la libertad va directamente en contra de la intersubjetividad teórica que podría requerir el determinismo para no ser considerado un constructo idealista. Esto se debe a la idea determinista de alcanzar la explicación 
objetiva de la realidad desde un único principio. Esto se pone en evidencia cuando observamos que el determinismo, asumiendo la validez de la causalidad lógico-fisicalista como algo evidente y autoexplicativo, pone en duda la validez de la libertad humana por medio de este principio en (a) y (a'), y pasa inmediatamente a certificar la inexistencia de tal libertad sin más apoyos teóricos en (b) y (b'). De este modo, el determinismo no solo merma su potencial explicativo sino que, a la vez, elimina la propiedad que podría mantenerlo en relación con las ciencias experimentales, es decir, la comunicabilidad con los principios teóricos de las otras ciencias que abre la racionalidad hacia la intersubjetividad como característica esencial para alcanzar una cierta fiabilidad.

\section{Conclusión}

El debate sobre la existencia de la libertad humana es una de las discusiones contemporáneas más complejas en la filosofía analítica. Su fundamento se encuentra en la admisión del determinismo como una teoría que podría ser fiable. Sin embargo, en este artículo, hemos demostrado la imposibilidad de que el determinismo pueda cumplir con su objetivo de erigirse como una teoría que pueda poseer una coherencia que la haga compatible con las ciencias experimentales.

La estrategia utilizada por el determinismo pretende que la duda sobre la existencia de la libertad humana se convierta en una certeza amparándose simplemente en el principio que rige su idea de visión científica del mundo. Sin embargo, tal como hemos indicado, el determinismo asume un supuesto poder autoexplicativo de la causalidad lógico-fisicalista que utiliza. Los presupuestos teóricos del determinismo llevan a que este se vea impedido de apoyarse significativamente en los principios de otras ciencias para sustentar sus conclusiones, incluidas las mismas ciencias experimentales. Con ello, el determinismo merma su capacidad teórica de relacionarse con las demás ciencias, evitado una apertura racional que podría llevarle a alcanzar la intersubjetividad que, de darse, podría mantenerlo en contacto con las demás ciencias. 
Para alcanzar estas conclusiones nos hemos apoyado en la explicitación de algunos principios sobre la capacidad explicativa y la fiabilidad de las teorías científicas que hace Mariano Artigas en su libro La mente del universo. Con esto esperamos cumplir con el objetivo de demostrar la importancia de esta obra filosófica, que puede contribuir a la disolución de un complejo debate como el analizado, poniendo en evidencia que el determinismo no puede ser considerado, bajo ningún aspecto, una teoría filosófica coherente con la realidad.

\section{Referencias}

Alexander, P., A. Schlegel, W. Sinnott-Armstrong, A. Roskies, P. U. Tse, and T. Wheatley. 2015. "Dissecting the Readiness Potential. An Investigation of the Relationship between Readiness Potentials, Conscious Willing, and Action.” In Surrounding Free Will. Philosophy, Psychology, Neuroscience, edited by A. Mele, 203-230. Oxford: Oxford University Press.

Artigas, M. 1999. La mente del universo. Pamplona: Eunsa. Publicado en inglés como The mind of the universe: understanding science and religion (Radnor, Penn: Templeton Foundation Press, 2000).

Balaguer, M. 2009a. "The Metaphysical Irrelevance of the Compatibilism Debate (and, More Generally, of Conceptual Analysis).” The Southern Journal of Philosophy 47:1-24.

-. 2009b. "Why there are no good arguments for any interesting version of determinism.” Synthese 168:1-21.

Campbell, J. K. 2011. Free Will. Cambridge. UK: Polity Press.

Churchland, P. 2011. Braintrust. New Jersey: Princeton University Press.

-. 2013. "The benefists of realising you're just a brain. An interview of Grahan Lawton.”

New Scientist. New Scientist Nov. 27:30-31. http://www.newscientist.com.

Clarke, R. 2014. “Freedom, Responsibility, and Omitting to Act.” In Libertarian

Free Will. Contemporary Debates, edited by D. Palmer, 107-123. Oxford: Oxford University Press.

Fischer, J. M. 2007. “Compatibilism.” In Four Views on Free Will, edited by E. Sosa, 44-84. Massachusetts: Blackwell Publishing.

-. 2010. "The Frankfurt cases: The moral of the stories.” Philosophical Review 119:315-336. 
Frankfurt, H. 1969. “Alternate Possibilities and Moral Responsibility.” The Journal of Philosophy 66:829-839.

Ginet, K. 2014. “Can an Indeterministic Cause Leave a Choice Up to the Agent?” In Libertarian Free Will. Contemporary Debates, edited by D. Palmer, 27-34. Oxford: Oxford University Press.

Hintikka, J. 1973. Time and Necessity. Oxford: Oxford Clarendon Press.

-. 1979. "Necessity, Universality and Time in Aristotle.” In Articles on Aristotle. 3. Metaphysics, edited by J. Barnes, M. Schofield y R. Sorabji, 108-124. London: Duckworth.

Hoefer, C. 2015. “Causal Determinism.” In The Stanford Encyclopedia of Philosophy, edited by E. N. Zalta. http://plato.stanford.edu/archives/fall2015/entries/determinism-causal/

Honderich, T. 2011. "Effects, Determinism, neither Compatibilism nor Incompatibilism, Consciousness.” In The Oxford Handbook of Free Will, $2^{\text {nd }}$ ed., edited by R. Kane, 442-456. Oxford: Oxford University Press.

Kane, R. 2007. “Libertarianism.” In Four Views on Free Will, edited by E. Sosa, 5-43. Massachusetts: Blackwell Publishing.

-. 2014. "New Arguments in Debate on Libertarian Free Will: Responses to Contributors.” In Libertarian Free Will. Contemporary Debates, edited by D. Palmer, 179-214. Oxford: Oxford University Press.

Libet, B., C. A. Gleason, E. Wright y D. Pearl, 1983. “Time of Conscious Intention to Act in Relation to Onset of Cerebral Activity (Readiness-potential). The Unconscious Initiation of a Freely Voluntary Act.” Brain 106:623-642.

Liz, Manuel. 2007. "El mundo físico y el mundo. Otros fisicalismos también son posibles.” En Pluralidad de la filosofía analítica, editado por D. P. Chico y M. Barroso, 95-128. Madrid-México: Theoria cum Praxi.

Murillo, J. I., y J. M. Giménez-Amaya. 2008. “Tiempo, conciencia y libertad: consideraciones en torno al experimento de B. Libet y colaboradores.” Acta Philosophica 17:291-306.

O’Connor, T. 2002. “Libertarian Views: Dualist and Agent-Causal Theories.” In The Oxford Handbook of Free Will, edited by R. Kane, 337-355. Oxford: Oxford University Press.

-. 2011. "Agent-Causal Theories of Freedom.” In The Oxford Handbook of Free Will, $2^{\text {nd }}$ ed., edited by R. Kane, 309-328. Oxford: Oxford University Press.

-. 2014. "Free Will and Metaphysics.” In Libertarian Free Will. Contemporary Debates, edited by D. Palmer, 27-34. Oxford: Oxford University Press.

Pink, T. 2004. Free Will. A Very Short Introduction. Oxford: Oxford University Press. 
Sellars, W. 1966. “Fatalism and Determinism.” En Freedom and Determinism, editado por K. Lehrer, 141-174. New York: Random House.

Strawson, P. F. 1962. "Freedom and Resentment." Proceedings of the British Academy 48:187-211.

Suhler, C. y P. Churchland. 2011. “Control: conscious and otherwise.” Trends in Cognitive Sciences 8:341-347.

Van Inwagen, P. 1983. An Essay on Free Will. Oxford: Oxford University Press.

-. 1998. “The Mystery of Metaphysical Freedom.” In Metaphysics: The Big Questions, edited by P. Van Inwagen y D. Zimmerman. Oxford: Blackwell.

Vihvelin, K. 2011. “Arguments for Incompatibilism.” In The Stanford Encyclopedia of Philosophy, edited by E. N. Zalta. http://plato.stanford.edu/entries/incompatibilism-arguments/.

Wegner, D. 2002. The Illusion of Conscious Will. Cambridge Massachusetts: MIT Press. Wiggins, D. 1987. “Towards a Reasonable Libertarianism.” In Needs, Values, Truth. Essays in the Philosophy of Value, 269-302. London: Oxford University Press.

Zimmerman, M. 1997. “Moral Responsibility and Ignorance.” Ethics 107:410-426. 